\title{
PREPARATION AND SOME PROPERTIES OF CARBON NANOTUBES
}

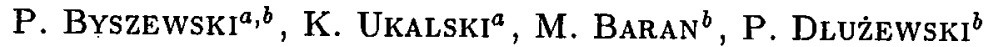 \\ AND M. KozŁOWSKI ${ }^{a}$ \\ a Institute of Vacuum Technology, Długa 44/50, 00-241 Warszawa, Poland \\ ${ }^{b}$ Institute of Physics, Polish Academy of Sciences \\ Al. Lotników 32/46, 02-668 Warszawa, Poland
}

Carbon nanotubes can be grown in the DC electric arc, they grow covered by a turbostratic graphite. The mechanism of the tubes growth is discussed. The magnetic susceptibility of samples, which contained aligned nanotubes exhibits anisotropic oscillatory behavior, which is ascribed to spatial quantization of the energy levels.

PACS numbers: 75.20.-g, 81.35.+k

\section{Introduction}

The carbon nanotubes attract much attention since Ijima [1] discovered that they were formed in the electric arc discharge between carbon electrodes. The first report on cylindrical carbon objects obtained in such process was published already in 1960 [2], however those tubes were of a macroscopic size and were not expected to exhibit any special physical properties. The diameter of Ijima's nanotubes on the other hand was of the order of several nanometers what implied a possible spatial localization of the electrons resulting from 1D structure. The length of nanotubes reaching microns suggested their possible application as nanowires [3].

There are different methods used to grow nanotubes. They can be grown either in the DC electric arc, e.g. [1], or AC, e.g. [4]. Often a catalytic method is applied when electrodes are doped with transition metals ( $\mathrm{Fe}, \mathrm{Co}$ ) [5] or by carbonization of hydrocarbons at high temperature and in electric field $[3,6]$. The method developed by Ebbesen et al. [7] (tubes were grown in the DC arc at very high temperature gradient and the amorphous carbon was burned out) seems to be the most promising one, for it allows one to obtain bundles of aligned nanotubes, free of amorphous or turbostratic carbon impurities ready for investigation of their physical properties.

The optical [8] or magnetic [9] measurements of powdered samples of nanotubes can be explained in terms of defected graphite without taking into account 
the low dimensions of the tubes, though the theoretical calculations $[10,11]$ predict that electronic properties of tubes ought to depend on their diameter. The energy bands of the tubes are discussed in terms of rolled graphene planes [11] when a limited size of the tubes imposed quantization on the $k_{x}$ component of the wave vector, while the $k_{y}$ component can change over the whole Brillouin zone. If the wave vector $k=\left(k_{x}, k_{y}\right)$ matches with a corner of the Brillouin zone, where the graphite conduction band touches the valence band, the tube energy gap is zero-metallic one, otherwise open-semiconducting one. These results resemble the HOMO-LUMO energy diagrams calculated for large elongated fullerenes [12]. The differences between experiment and effects expected from a theoretically calculated band structure result from the fact that the calculations were performed for idealized single shell, small diameter nanotubes, while the macroscopic samples contained multishell tubes of various sizes consisted of many graphene layers and were not aligned along their axis. Here we describe methods of preparation and evaluation of the quality of our samples and preliminary results of magnetic anisotropy measurements performed on the material containing bundles of aligned nanotubes.

\section{Growth of nanotubes}

Following the previous experiments on tubes growth [4] and on plasma processes in a carbon arc [13], the processes were carried out in the DC arc generated between graphite rods of $6 \mathrm{~mm}$ diameter, in helium atmosphere at pressure of about $0.6 \mathrm{~atm}$. and electric current of $80 \mathrm{~A}$. Similarly to [7], during the discharge the cathode was cooled (here) by a controlled flow of helium. At low electric current the growth rate of the carbon deposits was of the order of $3 \mathrm{~mm} / \mathrm{min}$ (evaporation rate $1.3 \mathrm{mg} / \mathrm{s}$ ). During the process almost $70 \%$ of carbon vaporized from the anode condensed on the cathode. A cross-section of the carbon deposits consists of three regions of different morphology and crystalline structure. The hard outer part built of the turbostratic graphite contains a region of a needle-like material aligned along the cathode axis (Fig. 1a). While increasing radial temperature gra-

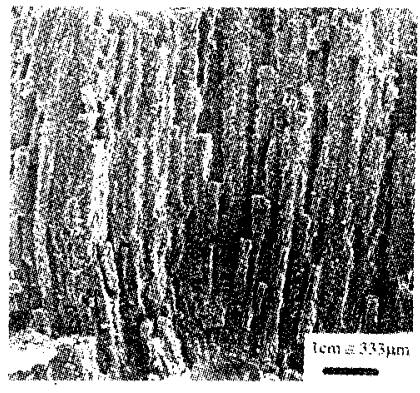

(a)

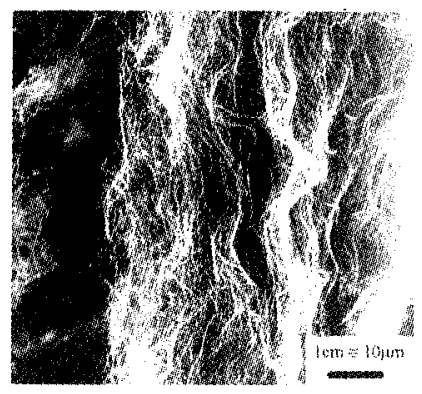

(b)

Fig. 1. The electron microscope image of the needles-like deposit, (a) before and (b) after heat treatment. 
dient by the high helium flow, needles start to grow tilted towards the axis of the electrode where they melt to form a crystalline graphite region. The needles contain bundles of the nanotubes. The needles start to grow from a thin layer of the graphite deposited on the cathode at the very beginning of the discharge, probably they form seeds, further the growth of tubes simply requires the proper temperature gradient and a continuous supply of carbon clusters for crystallization process. This in turn requires a stabilized DC power supply because of the low heat capacity of electrodes, the AC (or pulsed DC) discharge is insufficient to support continuous sublimation of the anode [13]. The temperature of the anode is balanced while heating by the electrons and cooling by carbon sublimation (apart from radiation, thermal conductivity and convection), when the heat transferred to the cathode by condensing carbon has to be dissipated by thermal conductivity, radiation and carbon resublimation. In fact, it seems that the cathode heats up along the whole deposit. In the carbon deposits only the species of high thermal conductivity, connected to the heat sink, i.e. cooled base or outer shell, can survive. To these belong tubes not ribbons or whiskers with weakly bound atoms at their edges.

The turbostratic graphite, contained also in the needles, can partially originate from overheated, melted tubes. The turbostratic graphite covering nanotubes could be burned out [7] if the needle-like material was heat treated in air at slowly rising temperature at the rate of $3.6 \mathrm{deg} / \mathrm{min}$ to $690^{\circ} \mathrm{C}$. During this process most of the mass (also the thinnest tubes) is lost leaving a network of carbon tubes (Fig. 1b).

\section{High resolution transmission electron microscopy images (HRTEM)}

The morphology of the samples at various stages of the heat treatment was verified if the network consisted of tubes or graphite fibers. It was found that thick fibers of Fig. 2 split into separate objects, which could be imaged by HRTEM and all the fibers checked revealed the tube structure. Because in the samples prepared in this way we could nowhere find any electron diffraction pattern characteristic of crystalline graphite, and all objects, which could be directly imaged, showed tubes structures, therefore we believe that the whole macroscopic samples consisted of multishell tubes (Fig. 2). The average radius of the tubes ranges from $r_{\mathrm{t}}=30$ to $50 \AA$. The electron diffraction of the bundle of tubes was used to determine their morphology (Fig. 3). The diffraction shows some misorientation of the tubes and the distances scaled to $\mathrm{C}-\mathrm{C}=1.42 \AA$ : the interlayer distance $d=3.56 \AA$, cross hexagons distances of $1.23 \AA$ and $2.13 \AA((11)$ and (10) graphite planes, respectively). There is a noticeable sixfold symmetry at (10) reflex which points to a preferential orientation of the carbon hexagons. It is unexpected, because to form a multishell tube of $60 \AA$ in diameter, graphene planes can be rolled with various helicity [14]. However high abundance of the $\mathrm{C}_{2}$ complexes in the thermal plasma [13], together with the complexes attachment energy favors the $\langle 1,1\rangle$ growth direction of the graphene planes because then each attached $\mathrm{C}_{2}$ complex completes a hexagon. Using the notation after [10] for the tubes, it corresponds to the uniform growth of the $(n, n)$-type tubes or a sawtooth growth mode of the $(n, 0)$-types. 
Fig.2

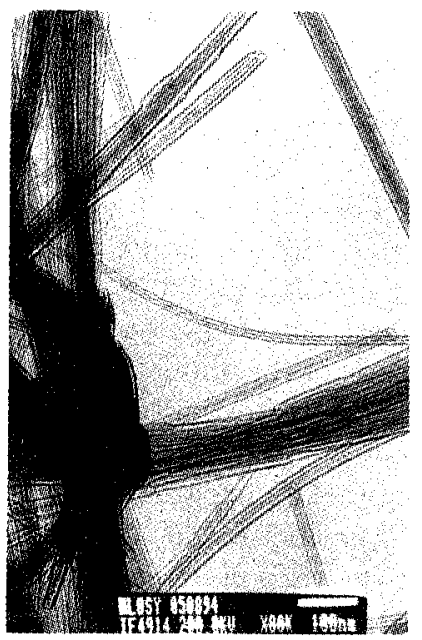

Fig. 2. The HRTEM image showing tubes in the samples.

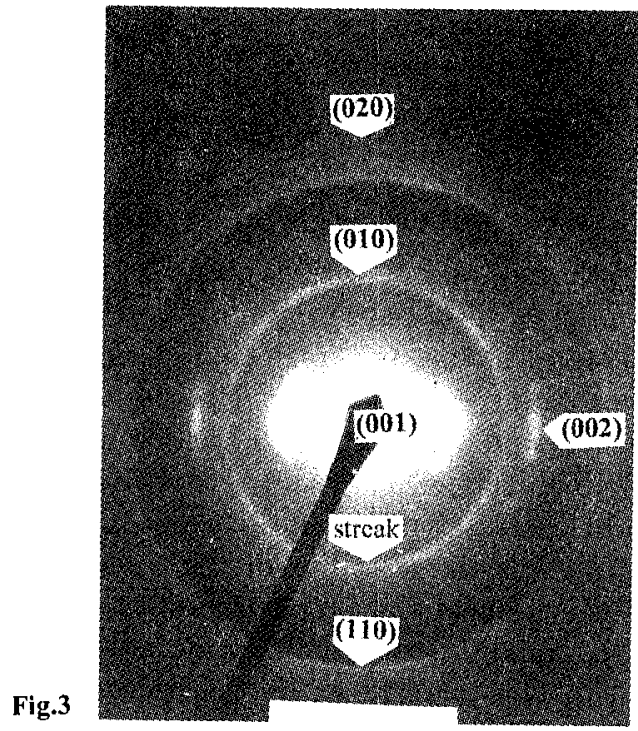

Fig. 3. The electron diffraction pattern of a bundle of nanotubes. The streaks show that some graphene planes are aligned in the $\langle 2,1\rangle$ direction along the tubes axes.

The non uniform distribution of (100) reflexes and their rotation relative to the tube axis suggest that they might originate from $(\approx 9 n, n)$-tubes, which had grown in the sawtooth mode, showing that the experimental conditions favored somehow their growth. 


\section{Magnetic susceptibility measurements}

The magnetic susceptibility of a sample containing axially aligned nanotubes was measured with the use of a SQUID magnetometer. Unfortunately, because of the problems with mounting of the sample in the sample holder (contribution to susceptibility of quartz holder is practically negligible), we were still unable to measure the pure tube sample, the one used for the magnetic measurements contained turbostratic graphite therefore the results presented in Fig. 4 will be discussed only qualitatively. The susceptibility $\chi_{\perp}$ perpendicular and $\chi_{\|}$parallel of the average tubes direction to the magnetic field evidently differs, the variation with the temperature is stronger than for graphite [15] or tubes [9], there is also oscillatory behavior seen at approximately $40 \mathrm{~K}$ in $\chi_{\|}$. The position of this feature does not depend on the magnetic field though its amplitude increases with the field.

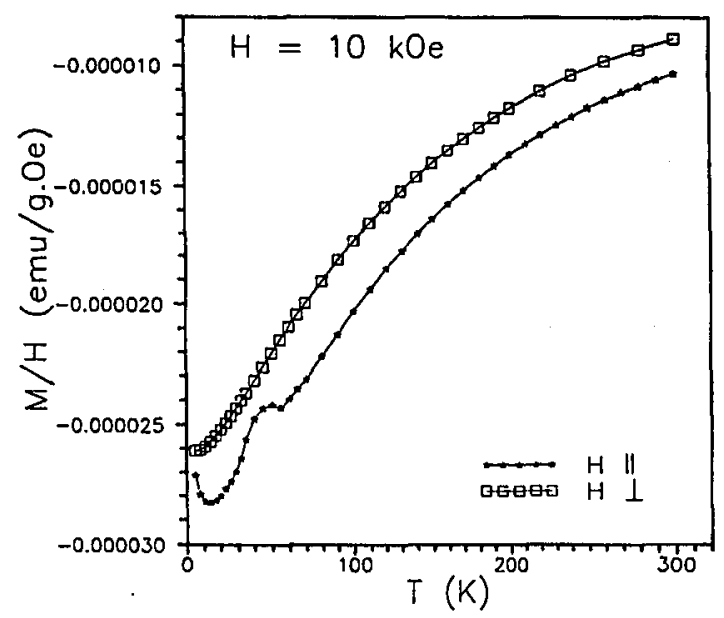

Fig. 4. The susceptibility $\chi$ of the sample, which still contained turbostratic graphite.

The susceptibility of graphite depends on the degree of graphitization [15] and for pure, well crystallized one, at room temperature, reaches a value about $\chi=-20 \div-30 \times 10^{-6} \mathrm{emu} / \mathrm{g}$ Oe, for the turbostratic graphite $\chi$ rises to $-35 \times$ $10^{-6} \mathrm{emu} / \mathrm{g}$ Oe. With lowering temperature $|\chi|$ increases by a factor of three, what is explained in terms the of the graphite band structure where the diamagnetism originates in the increase in the total energy associated with the rearrangement of free carriers. The susceptibility also depends on doping.

The magnetic field applied in these experiments falls into the low/average magnetic field region for free carriers in graphite, if the magnetic length $\left(\lambda_{H}=\right.$ $\sqrt{(h / 2 \pi e H)},\left(\lambda_{1 T}=256 \AA\right)$ describing the localization of a carrier in the plane perpendicular to the magnetic field is compared to the tubes diameter, i.e. the first cyclotron orbit radius $r_{\mathrm{c}}=\lambda_{H}$ here is larger than the tubes diameter. The energy of the lowest Landau level $E_{\mathrm{L}}=h \omega_{\mathrm{c}} / 2=h^{2} /\left(m \lambda_{H}^{2}\right)$ depends on the magnetic 
field and for $H=1 \mathrm{~T}$, the free carriers effective mass in graphite $m_{\mathrm{c}}^{*}=0.06 \mathrm{~m}$ and $m_{\mathrm{h}}^{*}=0.04 m$ [15] is $E_{\mathrm{L}}=0.97 \mathrm{meV}$ and $1.4 \mathrm{meV}$ for electrons and holes, respectively. The oscillation in $\chi_{\|}$cannot be connected with any of the magnetic oscillatory effects because its position does not vary with $H$. In a crude model of an electron of mass $m_{\mathrm{e}}^{*}$ in a potential well of the size of the tube radius $r_{\mathrm{t}}=50 \AA$ one gets higher energy $E_{w}=40 \mathrm{meV}$ (the same particle in a smaller well), which is independent of $H$ and is to be scaled by the mass, because we doubt that an effective mass approximation holds for tubes containing 100 graphite hexagons in their circumference.

We are therefore inclined to ascribe the oscillatory effect to the rearrangement of electrons at $40 \mathrm{~K}(k T=3.4 \mathrm{meV})$ on the spatially quantized energy levels and the magnetic field only probes the states.

\section{Free carriers motion on carbon tube}

The energy of free electrons located on the tubes can be discussed in terms of the model of defected graphite plane. If the tube radius is large, the behavior of free carriers located on the carbon nanotube does not differ from carriers in graphite, in a two-dimensional approach. For carbon tube extended in $z$ direction, the $k_{z}$ component of a carrier is not affected by the deformation introduced during rolling the flat plane, but now carriers can move in $x-y$ plane. Their behavior can be described by the deformation vector potential:

$$
\boldsymbol{A}=\hbar / r_{\mathrm{t}}^{2}[-y / 2, x / 2]
$$

and Hamiltonian

$$
H=(1 / 2 m)(\boldsymbol{p}+\boldsymbol{A})^{2},
$$

which satisfies the requirement of circular motion of the carrier with an effective mass $m$ on the orbit of the radius $r_{\mathrm{c}}=(2 n+1)^{1 / 2} r_{\mathrm{t}}$ with the "cyclotron" angular frequency $\omega_{\mathrm{c}}^{\mathrm{t}}=\hbar / m r_{\mathrm{t}}^{2}$ (in the ground state), the energy levels $E_{n}^{\mathrm{t}}=$ $(n+1 / 2) \hbar\left(h / m r_{\mathrm{t}}^{2}\right)$. The states are determined by the total angular momentum and its projection on the tube axes. The deformation potential secures the momentum exchange of a moving carrier with the network of the tube, momentum needed to compensate the deformation of the "lattice".

The degeneracy in respect to the projection of the angular momentum $\pm m$ is removed by the magnetic field parallel to the tube axes. The magnetic field with a potential of the form

$$
\boldsymbol{A}^{\prime}=I I[-y / 2, x / 2]
$$

splits the ground state to two with the corresponding angular frequencies $\omega_{\mathrm{c}}^{\mathrm{t}}+\omega_{\mathrm{c}}^{H}$ (diamagnetic) and $-\omega_{\mathrm{c}}^{\mathrm{t}}+\omega_{\mathrm{c}}^{H}$ (paramagnetic), where $\omega_{\mathrm{c}}^{H}=e H / m$. While the magnetic contribution $\omega_{\mathrm{c}}^{H}$ always increases the energy, the motion imposed by the tube $\omega_{\mathrm{c}}^{\mathrm{t}}$ can either increase or lower the energy. At high temperatures $\omega_{\mathrm{c}}^{\mathrm{t}} \tau \ll 1$ and $\omega_{\mathrm{c}}^{H} \tau \ll 1$ neither "tube" nor magnetic field quantization can be observed experimentally, at low temperatures when $\omega_{\mathrm{c}}^{\mathrm{t}} \tau \gg 1$, i.e. the scattering rate between the two states is very low, splitting by the magnetic field (even if $\omega_{\mathrm{c}}^{H} \tau<1$ ) may be seen experimentally. The sudden increase in $\kappa(T)$ (Fig. 4) at $T=40 \mathrm{~K}$ is 
tentatively ascribed to originate from the temperature induced transition from $\omega_{\mathrm{c}}^{\mathrm{t}} \tau \ll 1$ regime to $\omega_{\mathrm{c}}^{\mathrm{t}} \tau \gg 1$ at low temperatures. In high magnetic field when $\omega_{\mathrm{c}}^{H} \tau \gg 1$ and $\omega_{\mathrm{c}}^{\mathrm{t}} \tau<\omega_{\mathrm{c}}^{H} \tau$ the magnetic field contribution dominates carriers motion.

The work was partially supported by the grant N.2P 30225703 of the State Committee for Scientific Research (Republic of Poland).

\section{References}

[1] S. Ijima, Nature 354, 56 (1991).

[2] R. Bacon, J. Appl. Phys. 11, 283 (1960).

[3] R.E. Smalley, Mater. Sci. Eng. B 19, 1 (1993).

[4] P. Byszewski, P. Dłużewski, R. Diduszko, J. Mater. Res. 8, 118 (1993).

[5] D.S. Bethune, C.H. Klang, M.S. de Vires, G. Gorman, R. Savoy, J. Vazquez, R. Beyers, Nature 363, 605 (1993); S. Ijima, T. Ichihashi, Nature 363, 603 (1993).

[6] M. Endo, K. Takeuchi, S. Igarashi, K. Kobori, M. Shiraishi, H.W. Kroto, J. Phys. Chem. Solids 54, 1841 (1993).

[7] T.W. Ebbesen, P.M. Ajayan, Nature 358, 220 (1992).

[8] J. Kastner, T. Pichler, H. Kuzmany, S. Curran, W. Blau, D.N. Weldon, M. Delamesiere, S. Draper, H. Zandbergen, Chem. Phys. Lett., in print.

[9] Y. Murakami, T. Shibata, K. Okuyama, T. Arai, H. Suematsu, Y. Yoshida, J. Phys. Chem. Solids 54, 1861 (1993).

[10] J.W. Mintmire, D.H. Robertson, C.T. White, J. Phys. Chem. Solids 54, 1835 (1993).

[11] R. Saito, M. Fujita, G. Dresselhaus, M.S. Dresselhaus, Phys. Rev. B 46, 1804 (1992); Mat. Res. Soc. Proc. 247, 333 (1992).

[12] P.W. Fowler, J. Phys. Chem. Solids 54, 1825 (1993).

[13] H. Lange, A. Huczko, P. Byszewski, K. Ukalski, presented at $9^{\text {rd }}$ Eur. Congress on Thermal Plasma Processes, Aachen, 1994, to be published.

[14] N. Hamada, S. Sawada, S. Oshijama, Phys. Rev. Lett. 68, 1579 (1992).

[15] B.T. Kelly, Physics of Graphite, Applied Science Publishers, London, New Jersey 1981. 